Supporting Information

\title{
Reversible Transformations at Room Temperature among Three Types of CdTe Magic-Size Clusters
}

\author{
Qiu Shen ${ }^{\dagger}$, Chaoran Luan", Nelson Rowell ${ }^{\ddagger}$, \\ Meng Zhang ${ }^{\S}$, Kun Wang ${ }^{\S}$, Maureen Willis ${ }^{\zeta}$, Xiaoqin $\mathrm{Chen}^{*+}$, Kui $\mathrm{Yu}^{*+\S}$ \\ ${ }^{\dagger}$ Engineering Research Center in Biomaterials, Sichuan University, \\ 610065 Sichuan, P. R. China \\ "Laboratory of Ethnopharmacology, West China School of Medicine, \\ West China Hospital, Sichuan University, 610065 Sichuan, P. R. China \\ ${ }^{\ddagger}$ Metrology Research Centre, National Research Council Canada, \\ Ottawa, Ontario K1A 0R6, Canada \\ $\S$ Institute of Atomic and Molecular Physics, Sichuan University, \\ 610065 Sichuan, P. R. China \\ ¿School of Physical Science and Technology, Sichuan University, \\ 610065 Sichuan, P. R. China
}

X. C. (email: $\underline{x q c h e n @ s c u . e d u . c n)}$ or to K. Y. (email: kuiyu@scu.edu.cn) 


\section{Table of Contents}

Table S1 Explanation for technical terms used in our study

Table S2 Additional discussion of the substitution reactions proposed 4

Note S1 Additional discussion on reversible transformations of clusters 5

Note S2 Discussion on the disappearance of MSCs and the growth of QDs 6

Figure $\mathbf{S 1}$ Absorption of 11 samples from one room temperature reaction

Figure S2 Absorption of $60^{\circ} \mathrm{C} / 210 \mathrm{~min}$ sample $(30 \mu \mathrm{L})$ in two $3.00 \mathrm{~mL}$ Tol - OTA mixtures 8

Figure S3 Absorption of $60^{\circ} \mathrm{C} / 15 \mathrm{~min}$ sample $(30 \mu \mathrm{L})$ in three $3.00 \mathrm{~mL}$ Tol - OTA mixtures 9

Figure S4 Absorption of $60^{\circ} \mathrm{C} / 60 \mathrm{~min}$ sample $(100 \mu \mathrm{L})$ in two $3.00 \mathrm{~mL}$ Tol - OTA mixtures 10

Figure S5 Absorption of $60^{\circ} \mathrm{C} / 210$ min sample $(30 \mu \mathrm{L})$ in $3.00 \mathrm{~mL}$ OTA 12

Figure S6 Absorption of $60^{\circ} \mathrm{C} / 15 \mathrm{~min}$ sample $(30 \mu \mathrm{L})$ in two $3.00 \mathrm{~mL}$ Tol - BTA mixtures 13

Figure S7 Absorption of $50{ }^{\circ} \mathrm{C} / 120$ min sample $(50 \mu \mathrm{L})$ in three $1.00 \mathrm{~mL}$ Tol - BTA mixtures 14

Figure $\mathbf{5 8}$ Absorption of one RT/80 min sample $(200 \mu \mathrm{L})$ in three $3.00 \mathrm{~mL}$ Tol - BTA mixtures 15

$\begin{array}{lll}\text { Figure S9 Figure } 3 \text { spectra off-set in 2-dimension feature } & 16\end{array}$

Figure S10 Absorption of one RT/15 min sample $(25 \mu \mathrm{L})$ in 12 Tol - BTA - EtOH mixtures 17

Figure S11 Absorption of one RT/80 min sample $(200 \mu \mathrm{L})$ in ROH containing mixtures 19

Figure S12 All the spectra collected with partial shown in Figure $4 \quad 20$

Figure S13 Absorption of one RT/80 min sample $(50 \mu \mathrm{L})$ with OTA added to dispersions 21

Note S3 Additional discussion on the composition, configuration and size characterization of MSCs.

Table S3 Synthetic conditions for induction period samples 23

Table S4 Summary of the time interval $(\mathrm{min})$ for absorption spectrum collection 24 
Table S1 Explanation for technical terms used in our study.

\begin{tabular}{|c|c|c|}
\hline Acronym & Full name & Explanation \\
\hline-- & quasi isomerization & $\begin{array}{l}\text { a concept proposed for the transformation between } \\
\text { one PC and its counterpart MSC. }\end{array}$ \\
\hline PC & precursor compound & $\begin{array}{l}\text { a new product proposed which exists in one induction } \\
\text { period sample and is a MSC counterpart, with no } \\
\text { absorption at the MSC absorption peak position }\end{array}$ \\
\hline $\mathrm{Mo} / \mathrm{Fr}$ & monomer/fragment & $\begin{array}{l}\text { a fragment is larger, while a monomer was proposed } \\
\text { to be } \mathrm{Cd}_{2} \mathrm{E}_{2} \text {, where } \mathrm{E}=\mathrm{S}, \mathrm{Se} \text {, and Te } \\
\text { they form via the reaction of } \mathrm{Cd} \text { and } \mathrm{E} \text { precursors } \\
\text { or via } \mathrm{CdE} P C \text { fragmentation }\end{array}$ \\
\hline-- & configuration & shape, conformation, arrangement \\
\hline-- & composition & chemical composition, constitution, ingredient \\
\hline-- & absorption strength & optical density \\
\hline
\end{tabular}


Table S2 Additional discussion for the substitution reactions (Equations 2, 3 and 4 in the main text).

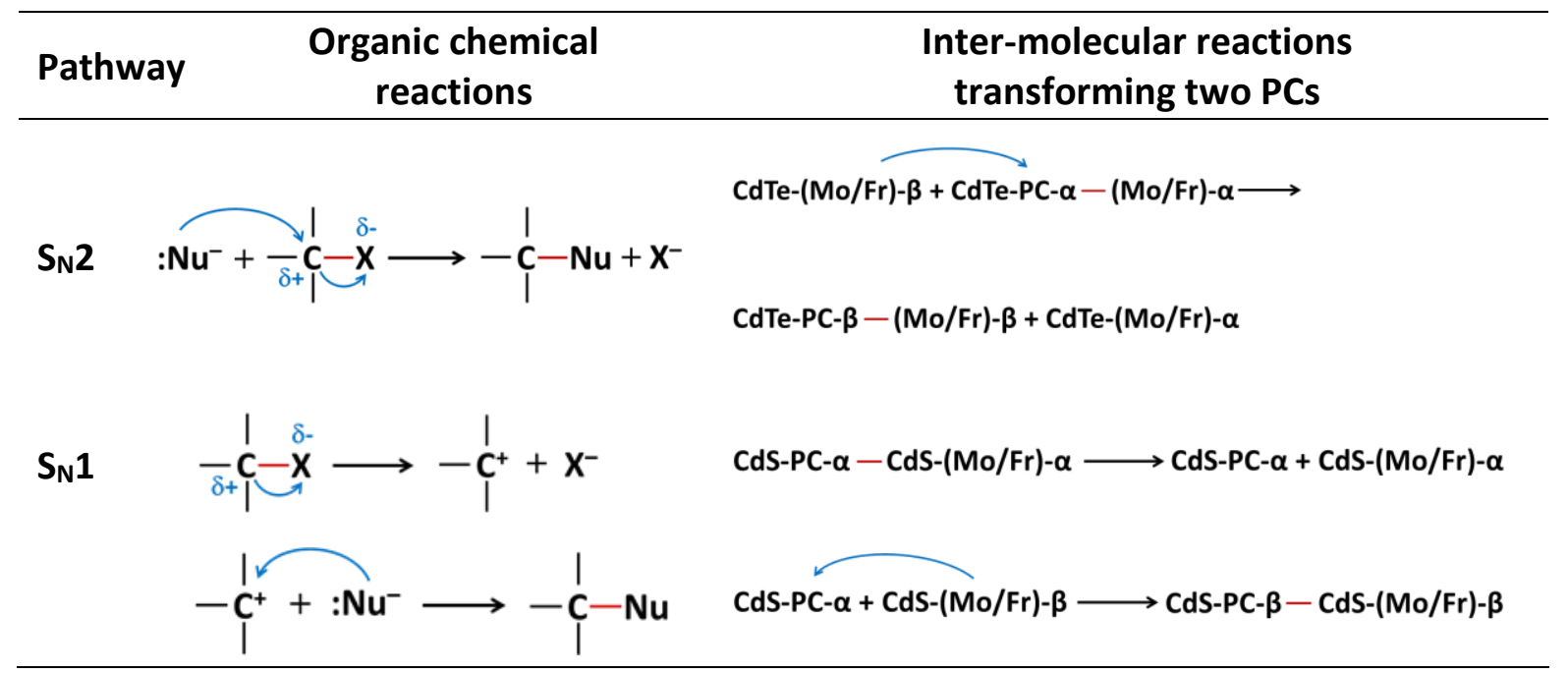

The possible pathways of the substitution reactions (Equations 2, 3 and 4 in the main text) have been analogous to the classical $\mathrm{S}_{\mathrm{N}} 2$ and $\mathrm{S}_{\mathrm{N}} 1$ reaction mechanisms, in which $\mathrm{S}$ stands for chemical substitution, $\mathrm{N}$ for nucleophilic, and the number for the kinetic order of the reaction. ${ }^{1}$

As in the $\mathrm{S}_{\mathrm{N}} 2$ reaction, a $\mathrm{PC}$ to $\mathrm{PC}$ transformation without a first-order reaction kinetics can be interpreted as follows: The addition of a substituent $\mathrm{CdTe}(\mathrm{Mo} / \mathrm{Fr}$ ) to the substrate $\mathrm{CdTe}$ $\mathrm{PC}$ takes place first; then, the CdTe (Mo/Fr) group leaves to result in the product CdTe PC.

As in the $S_{N} 1$ reaction, a PC to $P C$ transformation displaying a first-order reaction kinetics can be occurring as follows: A group of $\mathrm{CdS}(\mathrm{Mo} / \mathrm{Fr}$ ) leaves the substrate CdS PC first, the process of which is rate-determining, followed by the addition of a substituent $\mathrm{CdS}(\mathrm{Mo} / \mathrm{Fr}$ ) to result in the CdS PC product.

Furthermore, the presence of a protic agent, such as a primary amine and/or alcohol, has a significant influence on the substitution reactions that transform the two involved PCs. The effect of protic agents is not straightforward, however. The present study indicates the need for more theoretical and experimental effort to understand better such effects in the substitution reactions.

We also point out that limited, ligand-related studies have been discussed in the literature. For example, it has been shown that protic alcohols, such as $\mathrm{CH}_{3} \mathrm{OH}$ and $\mathrm{C}_{2} \mathrm{H}_{5} \mathrm{OH}$, probably assist in the desorption of carboxylate ligands. ${ }^{2}$ The existence of a complementary role for carboxylates and amines has been suggested by a computational study for tetrahedrally shaped CdSe magic clusters. ${ }^{3}$ Also, based on partial substitution of ligands, a transformation from $\mathrm{Au}_{25}$ to $\mathrm{Au}_{22}$ clusters has been reported. ${ }^{4}$

(1) J. P. Clayden, N. Greeves, S. Warren, P. D. Wothers, Organic Chemistry, Oxford University Press, Oxford, UK, 2001. 
(2) Hassinen, A.; Moreels, I.; De Nolf, K.; Smet, P. F.; Martins, J. C.; Hens, Z. Short-Chain Alcohols Strip X-Type Ligands and Quench the Luminescence of PbSe and CdSe Quantum Dots, Acetonitrile Does Not. J. Am. Chem. Soc. 2012, 134, 20705-20712.

(3) Mokkath, J. Computational Study of Magic-Size CdSe Clusters with Complementary Passivation by Carboxylic and Amine Ligands. J. Phys. Chem. C 2016, 120, 10015-10019.

(4) George, A.; Sundar, A.; Nair, A. S.; Maman, M. P.; Pathak, B.; Ramanan, N.; Mandal, S. Identification of Intermediate $\mathrm{Au}_{22}(\mathrm{SR})_{4}\left(\mathrm{SR}^{\prime}\right)_{14}$ Cluster on Ligand-Induced Transformation of $\mathrm{Au}_{25}(\mathrm{SR})_{18}$ Nanocluster. J. Phys. Chem. Lett. 2019, 10, 4571-4576.

Note S1 Additional discussion on reversible transformations of clusters.

Only recently, reversible isomerization has been reported for noble metal clusters, and transforming the two clusters reversibly was not that straighforward. ${ }^{1}$ The present study reports on reversible transformations that occur at room temperature between semiconductor CdTe MSCs, MSC-371, -417 , and -448. The three types of CdTe MSCs display optical absorption singlet peaking at 371, 417, and $448 \mathrm{~nm}$, respectively. In our two-step approach, the MSCs evolved when an induction period sample was dispersed in a mixture of toluene and a primary amine at room temperature. Many parameters and the interplay between them, which include the preparation temperature of the induction period sample, determine the evolution and the final predominant MSCs. For the forward transformation from CdTe MSC-448 to MSC-371 via MSC-417 to occur, the induction period sample needs to contain CdTe PC-448. For the backward transformation from CdTe MSC-371 to MSC-448 via MSC-417 to happen, CdTe PC-371 must be present the induction period sample. Our findings suggest that the reversible transformations are PC-enabled, which involve multiple steps such as quasi isomerization that transforms the PCs and their counterpart MSCs, as well as substitution reactions which transform the two involved PCs. ${ }^{2}$ It seems reasonable that the thermodynamic stability of one MSC is higher than that of its counterpart PC, also called an immediate PC. We propose that the added amines and/or alcohols may lead to ligand exchange; therefore, the PC involved MSC transformation occurs.

(1) Qin, Z.; Zhang, J.; Wan, C.; Liu, S.; Abroshan H.; Jin, R.; Li, G. Atomically Precise Nanoclusters with Reversible Isomeric Transformation for Rotary Nanomotors. Nat. Commun. 2020, 11, 6019.

(2) He, L.; Luan, C.; Rowell, N.; Zhang, M.; Chen, X.; Yu, K. Transformations Among Colloidal Semiconductor Magic-Size Clusters. Acc. Chem. Res. 2021, 54, 776-786. 
Note S2 Discussion on the disappearance of MSCs and the growth of QDs.

We have proposed that MSCs are transformed from their counterpart PCs. ${ }^{1}$ Also, we have demonstrated that the growth of CdS QDs at a relatively high reaction temperature results in the decrease in density of CdS MSC-311, as observed after a room temperature incubation for about one day. During the incubation, a PC to MSC-311 transformation occurred, while the decrease of the MSC-311 accompanied by the growth of the CdS QDs has been attributed to a fragmentation of the PC to monomers and fragments at the relatively high reaction temperature.

Furthermore, we have demonstrated that the TOPO-facilitated fragmentation of CdS PCS (after their formation) at a relatively low temperature leads to the nucleation and growth of small-size QDs with enhanced particle yield. ${ }^{2}$

Therefore, it is easy to know that our suggested pathway from the MSCs to monomers and fragments (via the PCs), ${ }^{1,2}$ which can be described as MSCs $\Leftrightarrow \mathrm{PCs} \Leftrightarrow$ monomers/fragments $\Leftrightarrow Q D s$, is consistent with what literature has claimed that MSCs might be a monomer source for the nucleation and growth of QDs. ${ }^{3,4}$

(1) Zhang, J.; Hao, X.; Rowell, N.; Kreouzis, T.; Han, S.; Fan, H.; Zhang, C.; Hu, C.; Zhang, M.; $\mathrm{Yu}, \mathrm{K}$. Individual Pathways in the Formation of Magic-Size Clusters and Conventional Quantum Dots. J. Phys. Chem. Lett. 2018, 9, 3660-3666.

(2) Li, L.; Zhang, J.; Zhang, M.; Rowell, N.; Zhang, C.; Wang, S.; Lu, J.; Fan, H.; Huang, W.; Chen, X.; Yu, K. Fragmentation of Magic-Size Cluster Precursor Compounds into Ultrasmall CdS Quantum Dots with Enhanced Particle Yield at Low Temperatures. Angew. Chem. Int. Ed. 2020, 59, 12013-12021.

(3) Jiang Z. J.; Kelley D F.; Role of Magic-sized Clusters in the Synthesis of CdSe Nanorods. ACS Nano 2010, 4, 1561-1572.

(4) Friedfeld, M. R.; Johnson, D. A.; Cossairt, B. M. Conversion of InP Clusters to Quantum Dots. Inorg. Chem. 2019, 58, 803-810. 

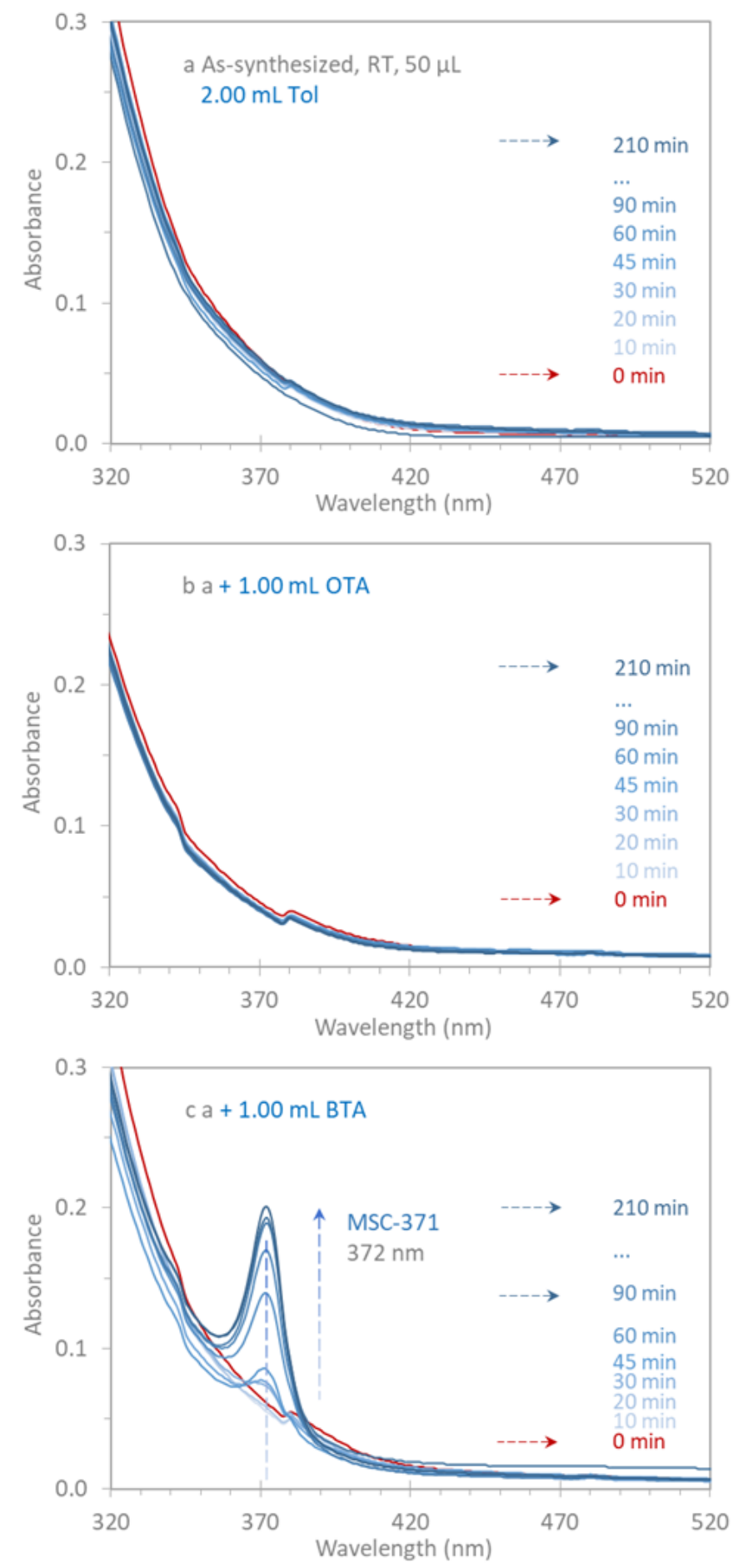

Figure S1. Optical absorption spectroscopy investigation for 11 samples extracted from one reaction within the prenucleation stage. The reaction was kept at room temperature and samples were taken at $0,10,20,30,45,60,90,120,150$, 180, and $210 \mathrm{~min}$. Each sample (50 $\mu \mathrm{L}$ ) was dispersed in $2.00 \mathrm{~mL}$ of Tol (a); after one spectrum was collected, $1.00 \mathrm{~mL}$ of OTA (b) or BTA (c) was added. In Tol (a) and in the Tol and OTA mixture (b), the 10 samples displayed featureless optical absorption. In the Tol and BTA mixture (c), the evolution of MSC-371 was observed for samples with the reaction periods of $30 \mathrm{~min}$ and longer. Thus, PC-371 formed increasing as the reaction proceeded. Temperature plays an important role for the PCs generated. This is understandable. For example, at relatively low temperatures, CdS PC311 and MSC-311 evolved; at

relatively high temperatures, CdS PC-322 and MSC-322 formed. ${ }^{1}$

(1) Zhang, B.; Zhu, T.; Ou, M.; Rowell, N.; Fan, H.; Han, J.; Tan, L.; Dove, M. T.; Ren, Y.; Zuo, X.; Han, S.; Zeng, J.; Yu, K. Thermally-Induced Reversible Structural Isomerization in Colloidal Semiconductor CdS Magic-Size Clusters. Nat. Commun. 2018, 9, 2499. 

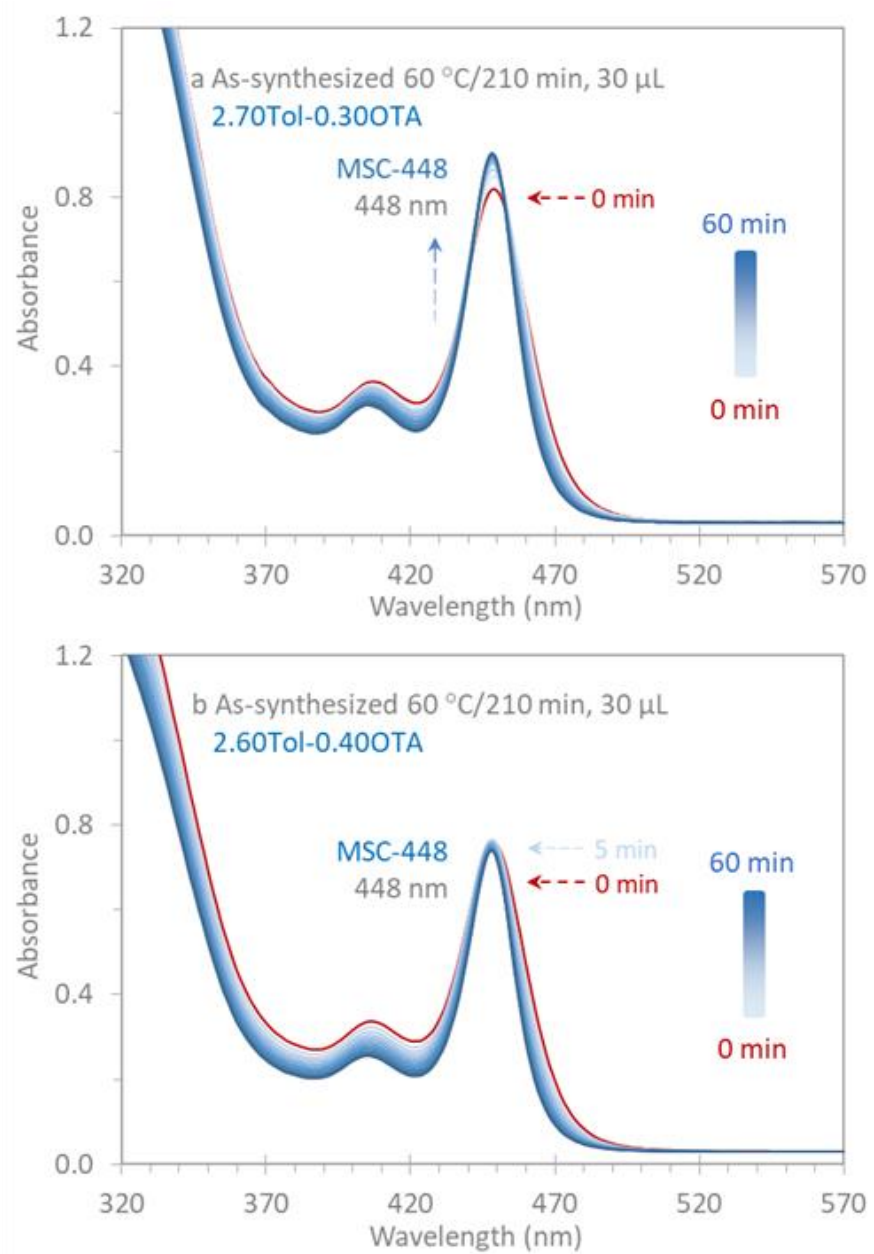

Figure S2. In situ optical absorption spectroscopy exploration of one as-synthesized sample $\left(60{ }^{\circ} \mathrm{C} / 210 \mathrm{~min}, 30 \mu \mathrm{L}\right.$ ) dispersed in $3.00 \mathrm{~mL}$ of Tol and OTA mixtures. In the two dispersions with $0.30 \mathrm{~mL}(\mathrm{a})$ and $0.40 \mathrm{~mL}$ of OTA (b), MSC-448 evolved immediately. In the former, the amount of MSC-448 increased monotonically in the first $20 \mathrm{~min}(\mathrm{OD}=0.84$ after the subtraction at $490 \mathrm{~nm}$ ) and changed little afterwards. In the latter, the amount of MSC-448 reached its maximum $(O D=0.72)$ at 5 min and then changed little. It seems that $0.30 \mathrm{~mL}$ OTA promoted more MSC-448 (via the transformation from PC-448, step 8 in Scheme 1) than $0.40 \mathrm{~mL}$ OTA did. 

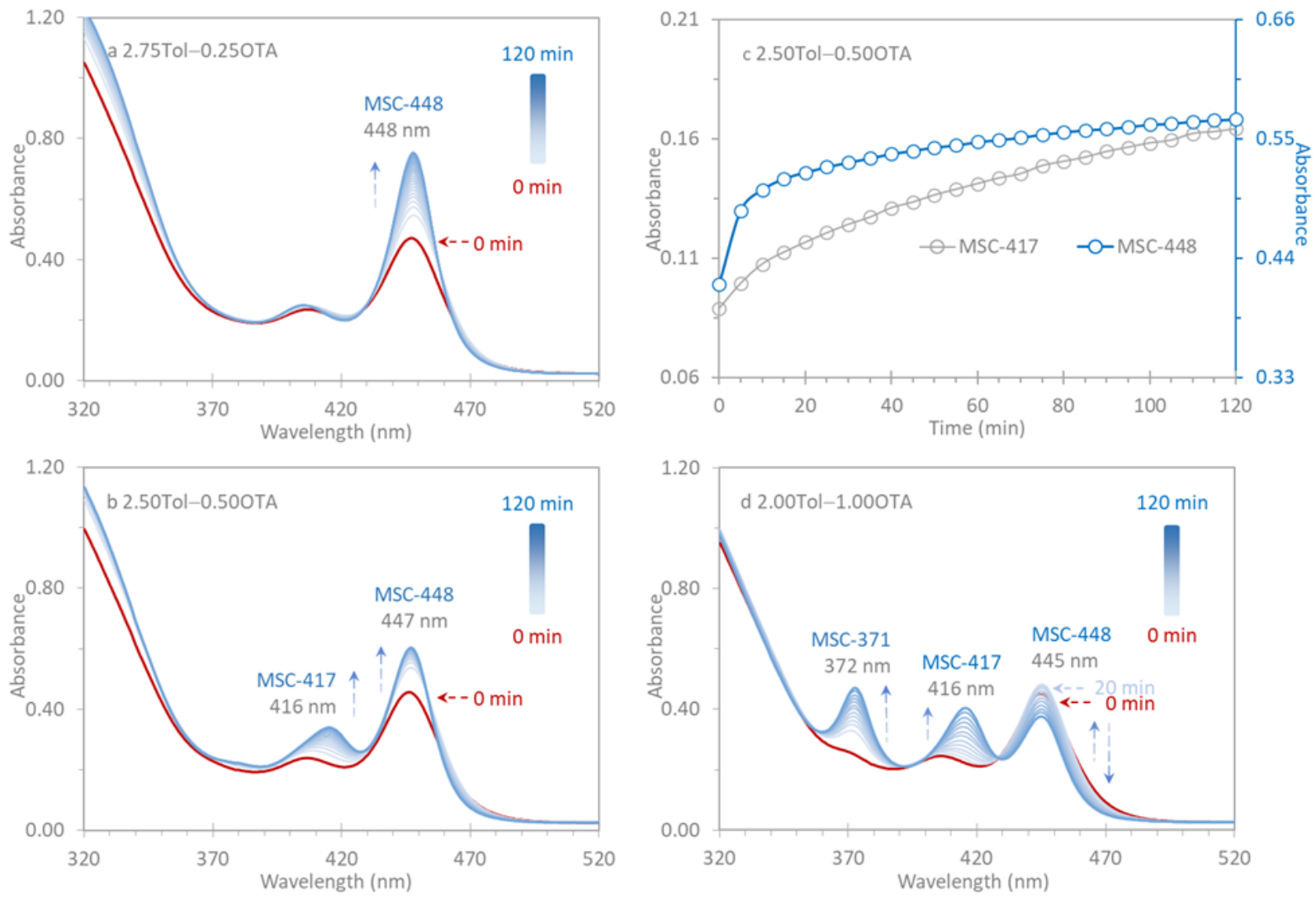

Figure S3. Time-resolved optical absorption spectra collected in situ from one induction period sample ( $30 \mu \mathrm{L}, 60^{\circ} \mathrm{C} / 15 \mathrm{~min}$ ) which was purified (and used for Figure 2 ). The sample was then placed in the $3.00 \mathrm{~mL}$ Tol-OTA mixtures as indicated, which contained 0.25 (a), 0.50 (b), and 1.00 (d) $\mathrm{mL}$ OTA, with the time-dependent absorbance for Dispersion b illustrated (c). The blue and gray dots are respectively for MSC-448 and MSC-417, with the blue and gray traces for visual guidance. The time interval for collection is $5 \mathrm{~min}(\mathrm{a})$ and $10 \mathrm{~min}$ (b and d). Parts $b$ and $d$ are presented in Parts $a$ and $b$ in Figure 2. In Part c, the absorbance at 448 and $417 \mathrm{~nm}$ (for the MSC-448 and MSC-417) was subtracted by that at $490 \mathrm{~nm}$, with addition subtraction at 417 contributed by MSC-448 for MSC-417. During 120 min period, the evolution rate for both MSC-417 (with OD from 0.09 to 0.16 ) and MSC-448 (with OD from 0.42 to 0.57 ) was faster in the initial stage and slowed down in the later stage. Accordingly, MSC-448 did not seem to be the direct reactant for MSC-417. The transformation to MSC-417 from MSC-448 should be via their corresponding PCs. 

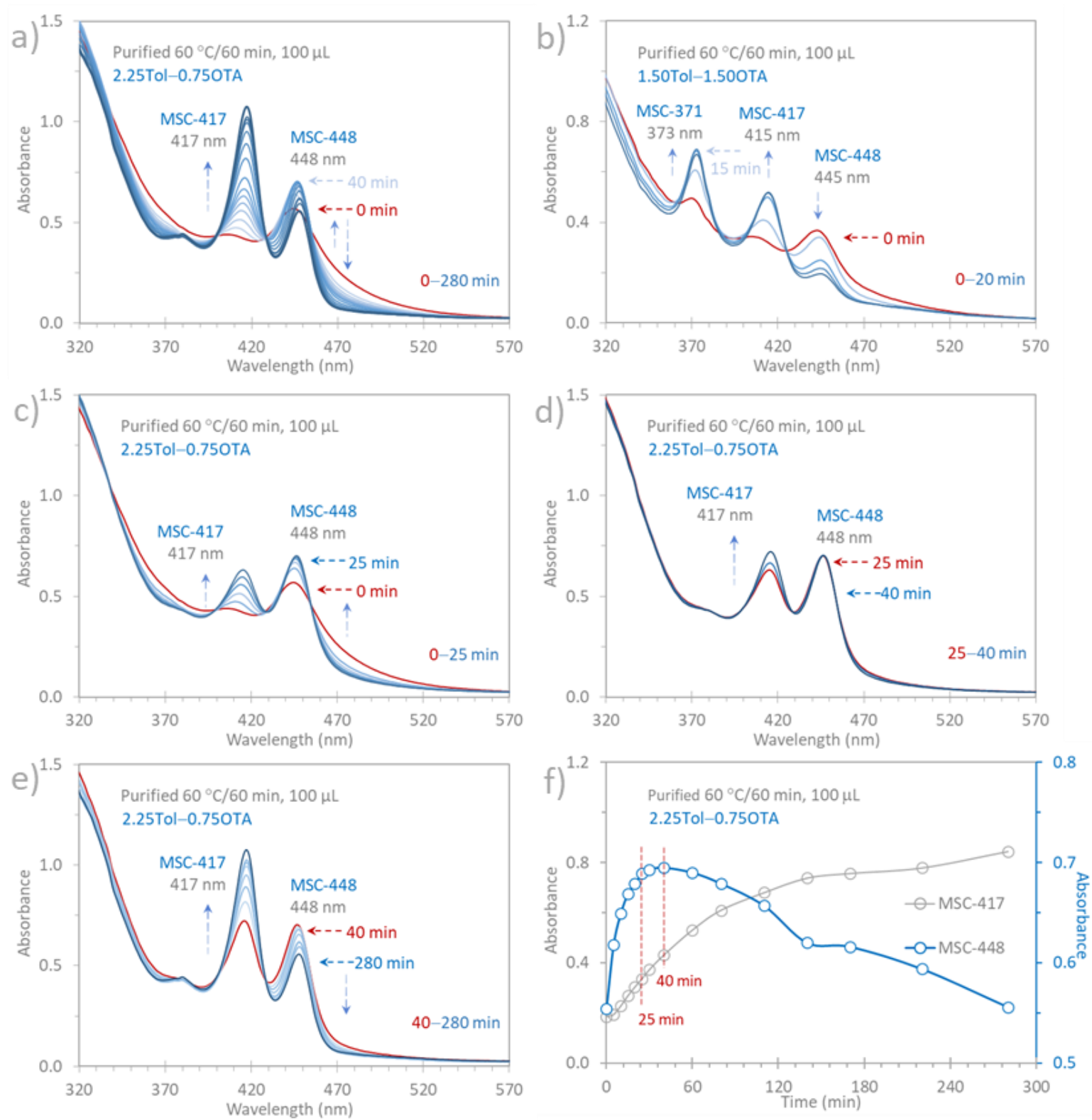

Figure S4. Time-resolved optical absorption spectroscopy (a-e) monitoring one induction period sample in dispersion $(3.00 \mathrm{~mL})$ and the corresponding time-dependent absorbance of MSCs (f) for Dispersion a. The sample $(100 \mu \mathrm{L})$ from one reaction $\left(60^{\circ} \mathrm{C} / 60 \mathrm{~min}\right)$ was purified and then dispersed in two Tol-OTA mixtures with 0.75 OTA (a) and $1.50 \mathrm{~mL}$ OTA (b). In Part a there are 15 spectra, with 0 to $25 \mathrm{~min}$ in c (collected every $5 \mathrm{~min}$ ), 25 to $40 \mathrm{~min}$ in d (collected at 25, 30, and $40 \mathrm{~min}$ ), and 40 to $280 \mathrm{~min}$ in e (collected at 40, 60, 80, 110, 140, 170,220 , and $280 \mathrm{~min}$ ). Part f illustrates the corresponding time-dependent absorbance of MSC-448 and MSC-417 for Dispersion a, with the blue and gray dots respectively for MSC448 and MSC-417, as well as the blue and gray traces for visual guidance. Evidently, the evolution of MSC-417 was not at the cost of MSC-448 only, but with other species, PC-417, consumed to produce MSC-417. In Part b there are 5 spectra collected every 5 min for the 0 
to $20 \mathrm{~min}$. In Dispersion a, MSC-448 was observed immediately ( 0 min, red trace), and increased to its maximum at $25 \mathrm{~min}$ and changed little till $40 \mathrm{~min}$ ( $\mathrm{c}$ and d); then, it decreased (e) in the following period. Monotonically, MSC-417 increased, with isosbestic point at $\sim 429 \mathrm{~nm}$. In Dispersion b, both MSC-448 and MSC-371 evolved immediately (0 min, red trace). MSC-371 and MSC-417 increased and reached their maximum at 15 and 20 min respectively, at the cost of the decrease of MSC-448; two isosbestic points were observed at $\sim 388$ and $\sim 428 \mathrm{~nm}$. Notably, the evolution of the MSC-417 did not follow first-order reaction kinetics. From 25 to 40 min (d), MSC-448 changed little, while MSC-417 apparently increased. Thus, the MSC-448 to MSC-417 transformations are PC-enabled, with step 9 towards PC-417 from PC-448 being rate-determining. 

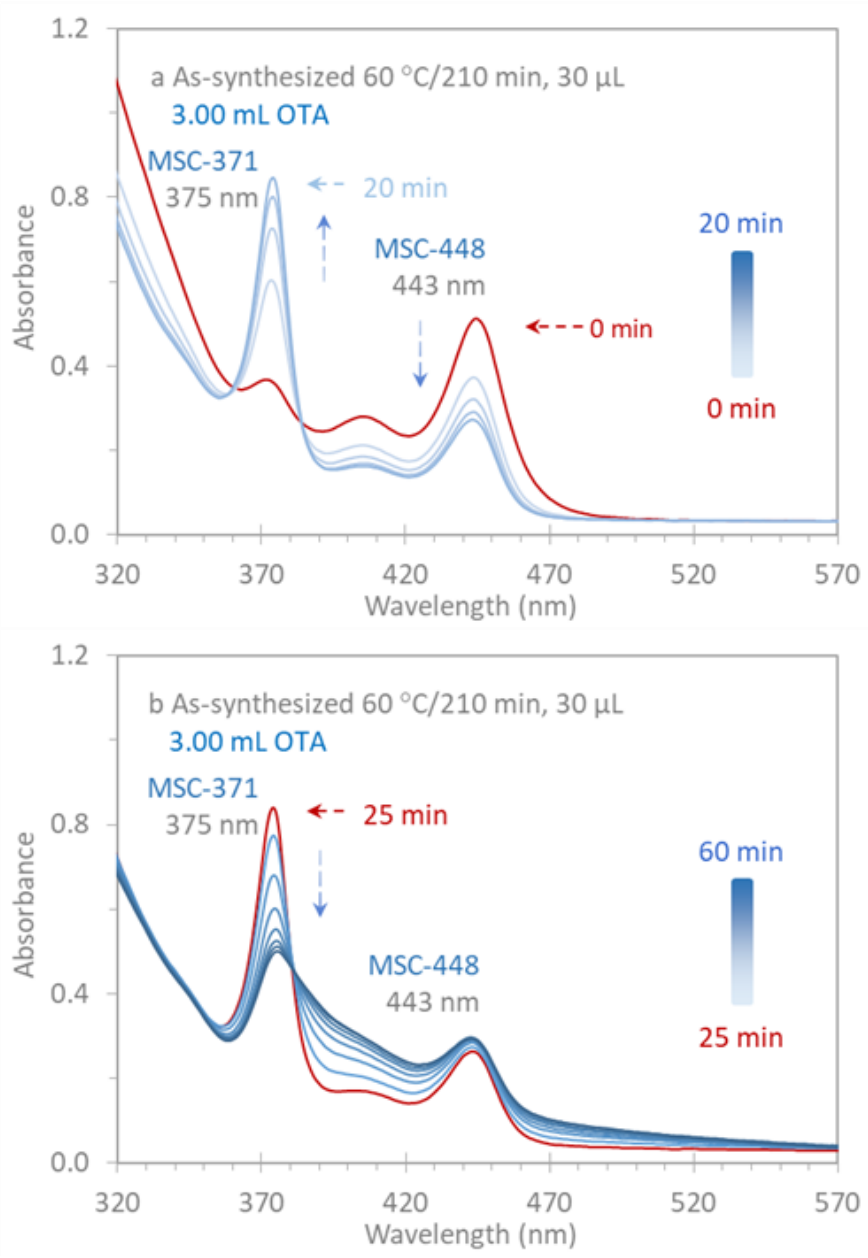

Figure S5. Optical absorption spectra collected in situ from one induction period sample $\left(60^{\circ} \mathrm{C} / 210 \mathrm{~min}, 30 \mu \mathrm{L}\right)$ in $3.00 \mathrm{~mL}$ of OTA. The spectra collected in the period of 0 to $60 \mathrm{~min}$ (every $5 \mathrm{~min}$ ). In Part a with those collected between 0 to $20 \mathrm{~min}$, MSC448 evolved immediately after dispersion (0 $\mathrm{min}$, red trace) and then decreased. MSC-371 increased continuously with an isosbestic points at $\sim 385 \mathrm{~nm}$. In Part b with spectra collected between 25 to $60 \mathrm{~min}$, MSC371 decreased, while MSC-448 changed little. 

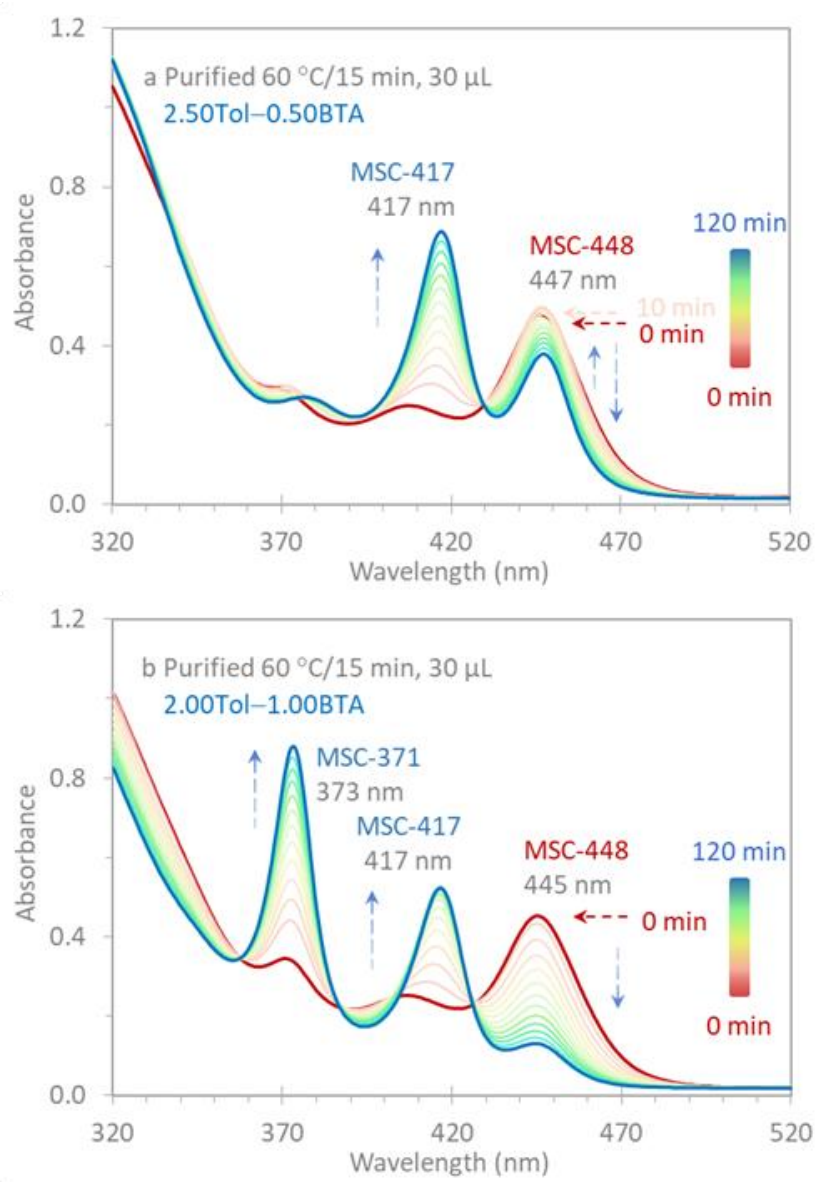

Figure S6. The partial spectra of those shown in Figures $2 \mathrm{c}$ and $2 \mathrm{~d}$. The spectra here had a time interval of $10 \mathrm{~min}$ (instead of $5 \mathrm{~min}$ ). Similarly, the evolution of MSC-417 (a) and that of MSC-371 and MSC-417 (b) are not only at the cost of MSC-448, but with their counterpart PCs involved as well. 

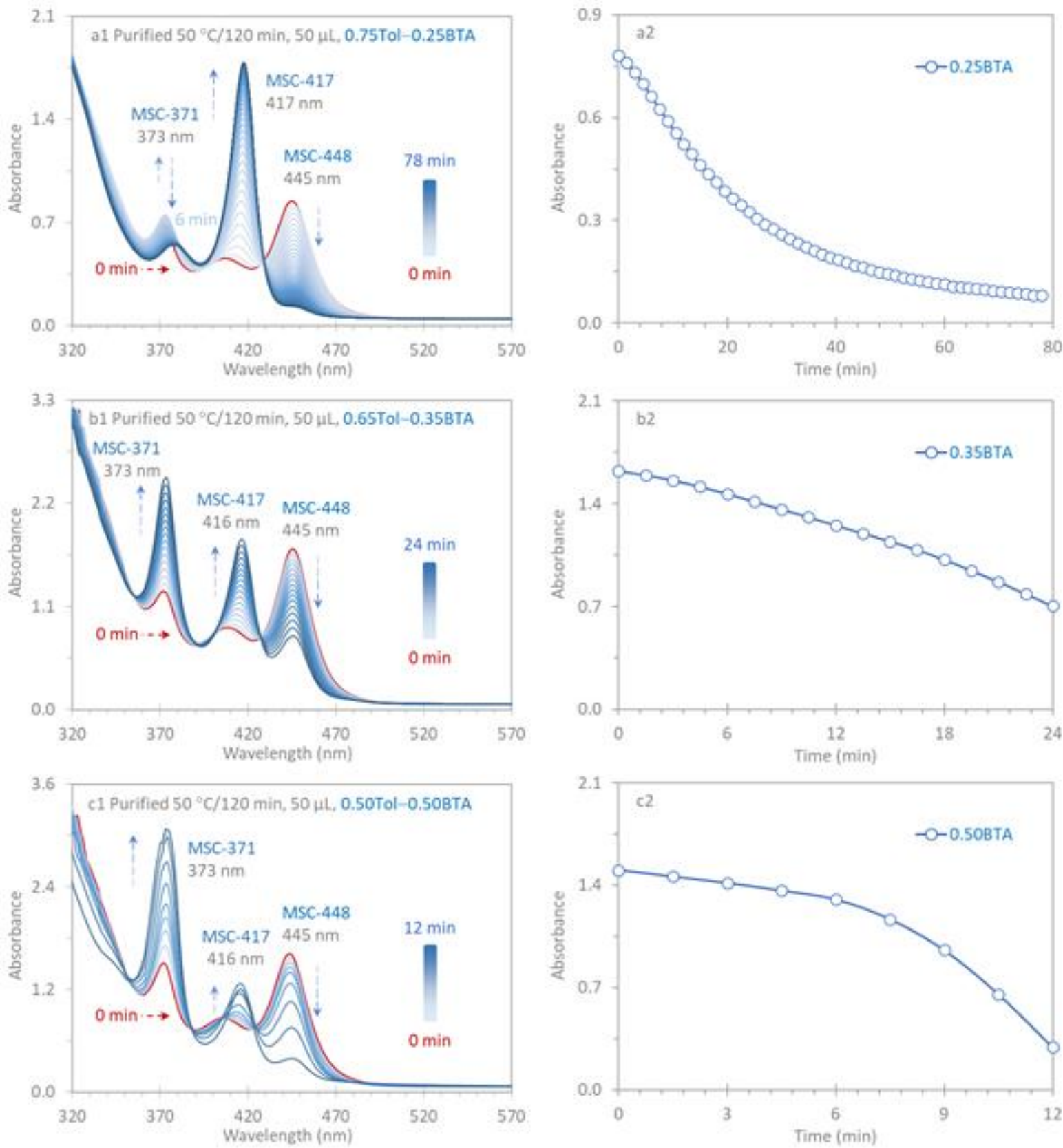

Figure S7. Optical absorption spectra and the collected in situ from one induction period sample $\left(50^{\circ} \mathrm{C} / 120 \mathrm{~min}, 50 \mu \mathrm{L}\right)$. The sample was purified and then dispersed in $1.00 \mathrm{~mL}$ of Tol and BTA mixtures containing 0.25 (a1), 0.35 (b1), and 0.50 (c1) $\mathrm{mL}$ of BTA (on the left panel). The spectra were collected every 1.5 min until 78 (a1), 24 (b1), and $12 \mathrm{~min}$ (c1). The corresponding time-dependent absorbance for MSC-448 is presented in Parts a2, b2, and c2 (on the right panel); the absorbance was subtracted by that at $490 \mathrm{~nm}$. In the left panel, both MSC-448 and MSC-371 were observed (0 min, red traces). In Dispersion a, MSC-371 increased to its maximum $(O D=0.69)$ at 6 min and decreased. MSC-448 decreased and 
MSC-417 increased, with an isosbestic point at $\sim 428 \mathrm{~nm}$. In Dispersion b, both MSC-371 and MSC-417 monotonically increased at a cost of the decrease of MSC-448, with two isosbestic points at 391 and $\sim 427 \mathrm{~nm}$. In Dispersion c, similar transformations (as those shown of Dispersion b) were observed but proceeded faster, with two isosbestic points at 389 and 425 nm. More MSC-371 and less MSC-417 were produced. More BTA promoted more step 10 via 9.
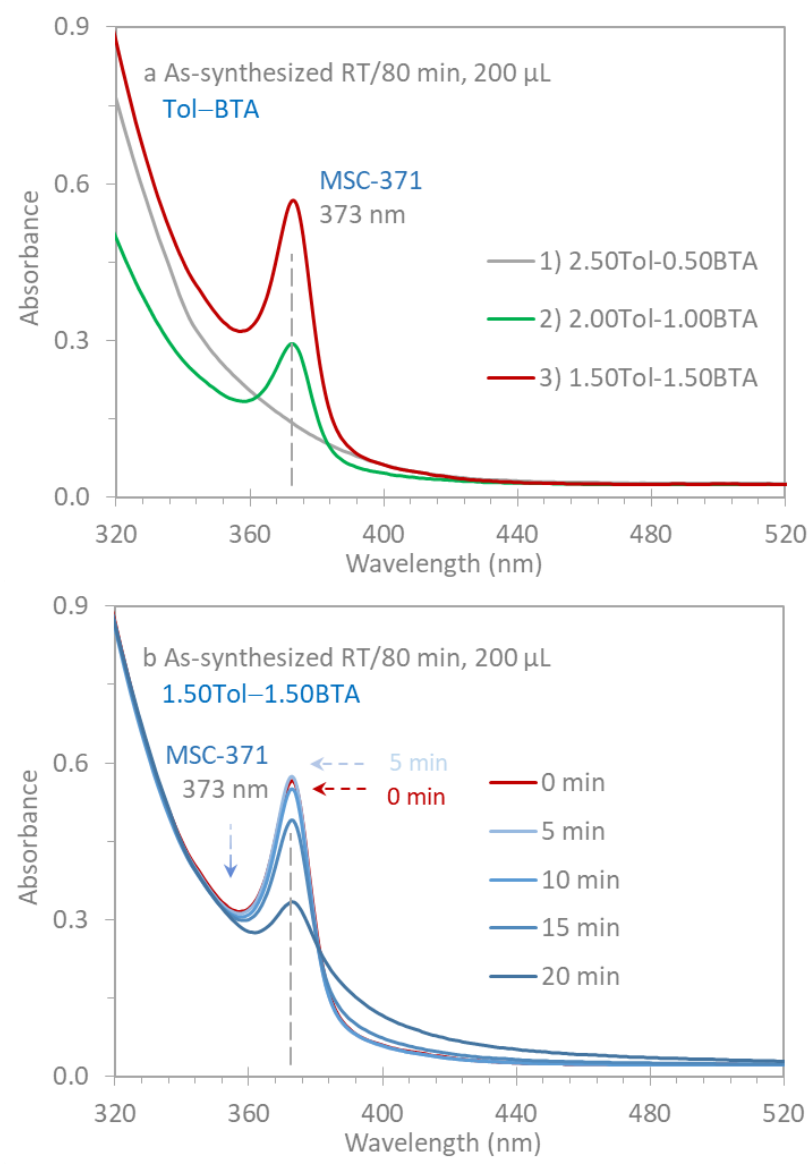

Figure S8. Optical absorption spectra of one induction period sample (from a room temperature reaction that proceeded for $80 \mathrm{~min}, 200 \mu \mathrm{L}$ ) in Tol and BTA mixtures $(3.00 \mathrm{~mL})$. In Part a, there are three spectra collected from the mixtures containing 0.50 (gray trace), 1.00 (green trace), and $1.50 \mathrm{~mL}$ (red trace) of BTA. The absorption is featureless with $0.50 \mathrm{~mL} \mathrm{BTA}$, while MSC371 evolved in the rest other two dispersions, with more in the mixture with $1.50 \mathrm{~mL}$ of BTA. In Part b, temporal evolution of absorption properties of the dispersion with $1.50 \mathrm{~mL}$ of BTA is shown with five spectra collected with an

interval of 5 min up to 20 min. MSC-371 evolved immediately, reaching its maximum at 5 min. Afterwards, it decreased gradually without evolution of other MSCs. Evidently, the small amount of BTA $(0.50 \mathrm{~mL})$ did not promote the PC-371 to MSC-371 transformation via step 1 in Scheme 1; furthermore, step 3 did not become active, namely the reaction from PC-371 to PC-417 to PC-448 (Equation 2) did not occur. 


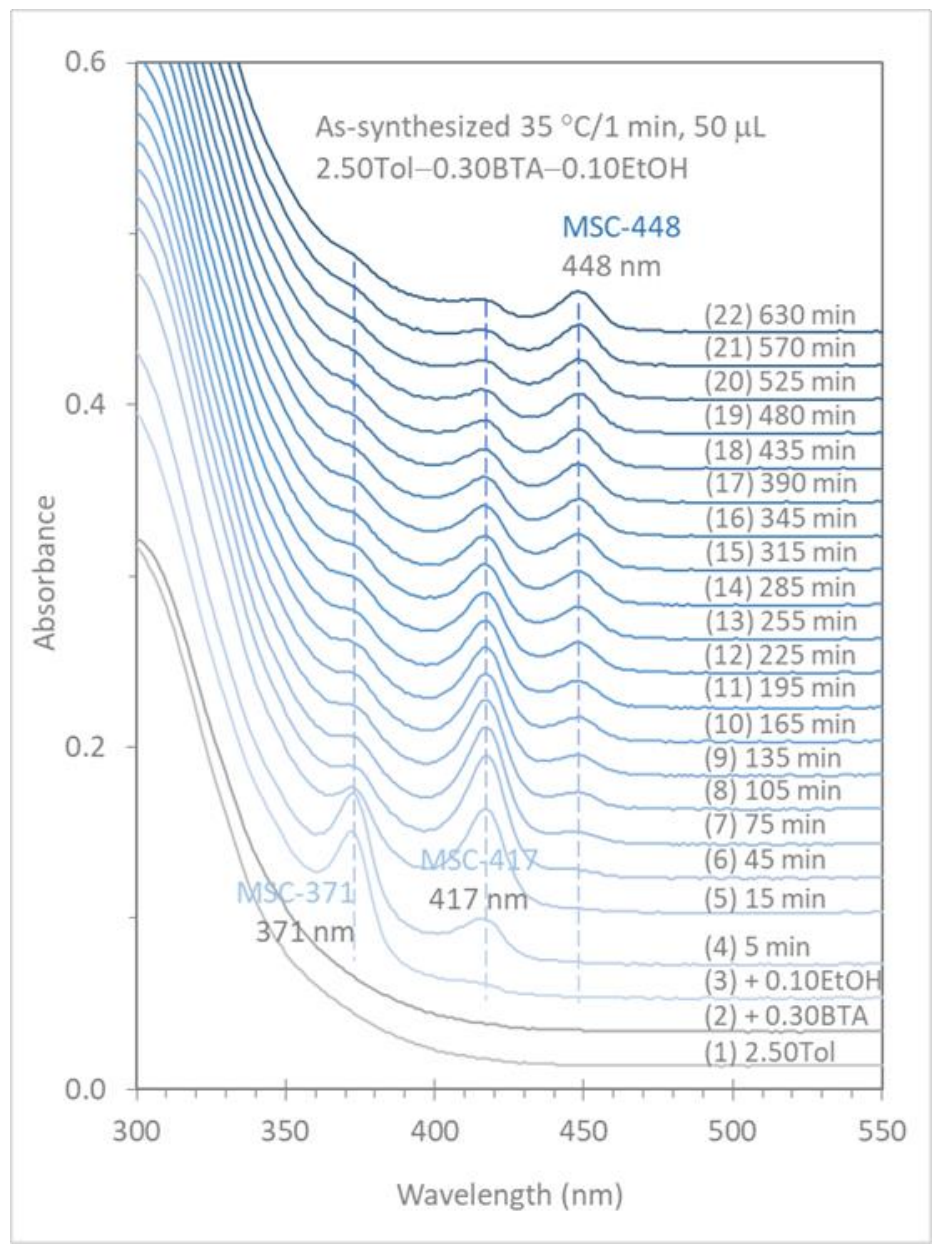

Figure S9. Optical absorption spectra shown in Figure 3, but plotted here with off set in a two-dimension feature. 

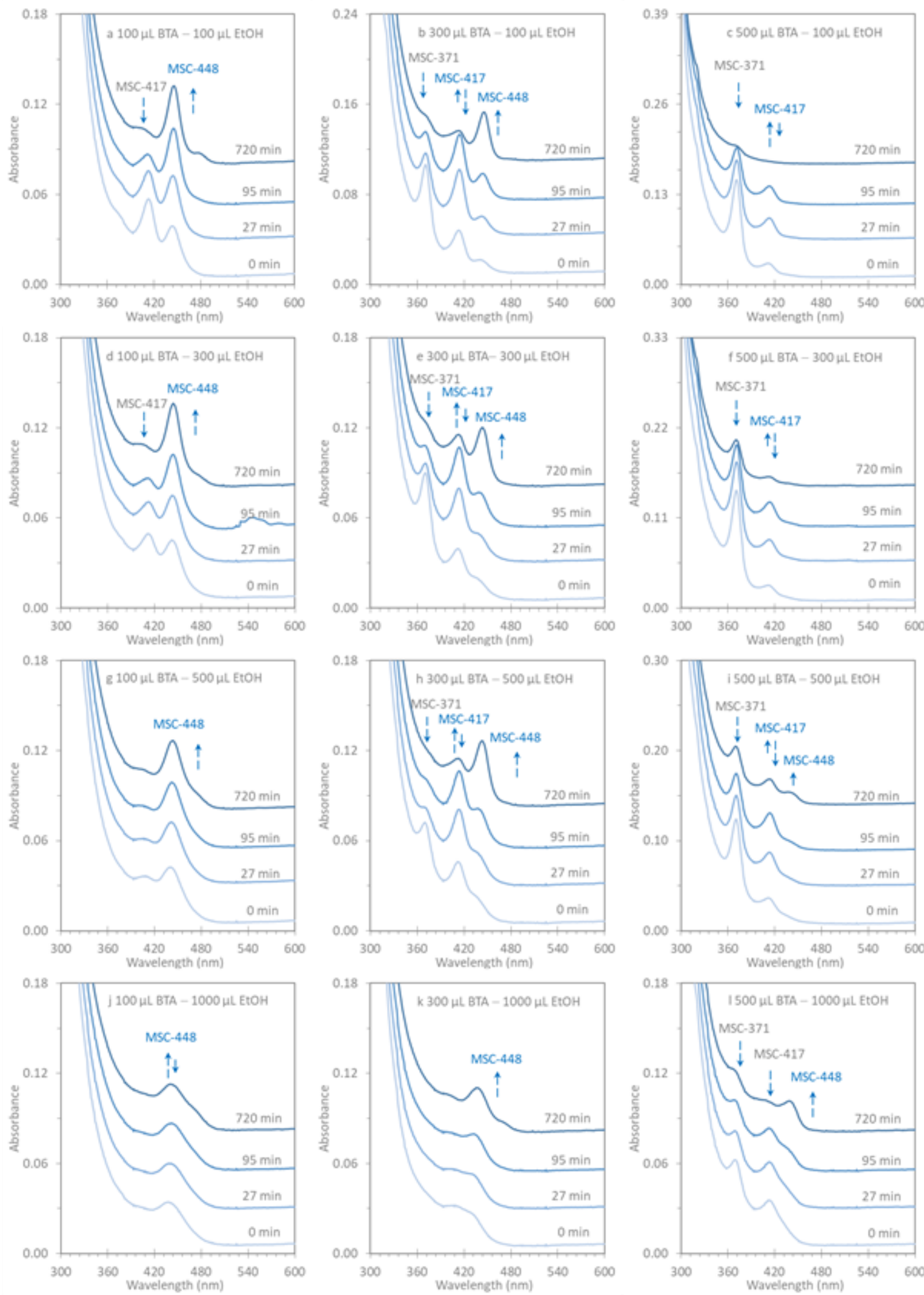

Figure S10. Optical absorption spectra collected from one as-synthesized sample (room temperature/15 min, $25 \mu \mathrm{L}$ ) in 12 dispersions. Each of the 12 dispersions had a total volume 
of $3.00 \mathrm{~mL}$, containing Tol, BTA, and EtOH. The volumes of BTA and ETOH are indicated. From the left to middle to right panels, the BTA amounts increase from 100, 300, and to $500 \mu \mathrm{L}$. From the top to bottom 4 panels, the EtOH amounts increase from 100, 300, 500, and to $1000 \mu \mathrm{L}$. For each of the 12 dispersions, there were 4 spectra collected at about $0,27,95$, and 720 min. Evidently, a larger amine amount favors MSC-371 (right panel), while a larger EtOH amount prefers MSC-448 (bottom panel).

For the left panel with the amount of BTA of $0.10 \mathrm{~mL}, \mathrm{MSC}-417$ and MSC-448 appeared first and the MSC-417 to MSC-448 transformations were observed with $0.10 \mathrm{~mL}$ (a) or 0.30 $\mathrm{mL}$ (d) EtOH; with $0.50 \mathrm{~mL}$ EtOH (g), MSC-448 appeared in the beginning and increased a tiny bit at $720 \mathrm{~min}$. With $1.00 \mathrm{~mL}$ of EtOH (j), the absorption signals broadened around 440 $\mathrm{nm}$. For the middle panel with $0.30 \mathrm{~mL}$ of BTA, the transformations from MSC-371 to MSC448 via MSC-417 was observed (b, e, and h). With $1.00 \mathrm{~mL}$ of EtOH (k), MSC-448 evolved. For the right panel with $0.50 \mathrm{~mL}$ of BTA, MSC-317 and MSC-417 evolved but display instability (c and f). With more EtOH ( $i$ and I), MSC-448 evolved as well. 

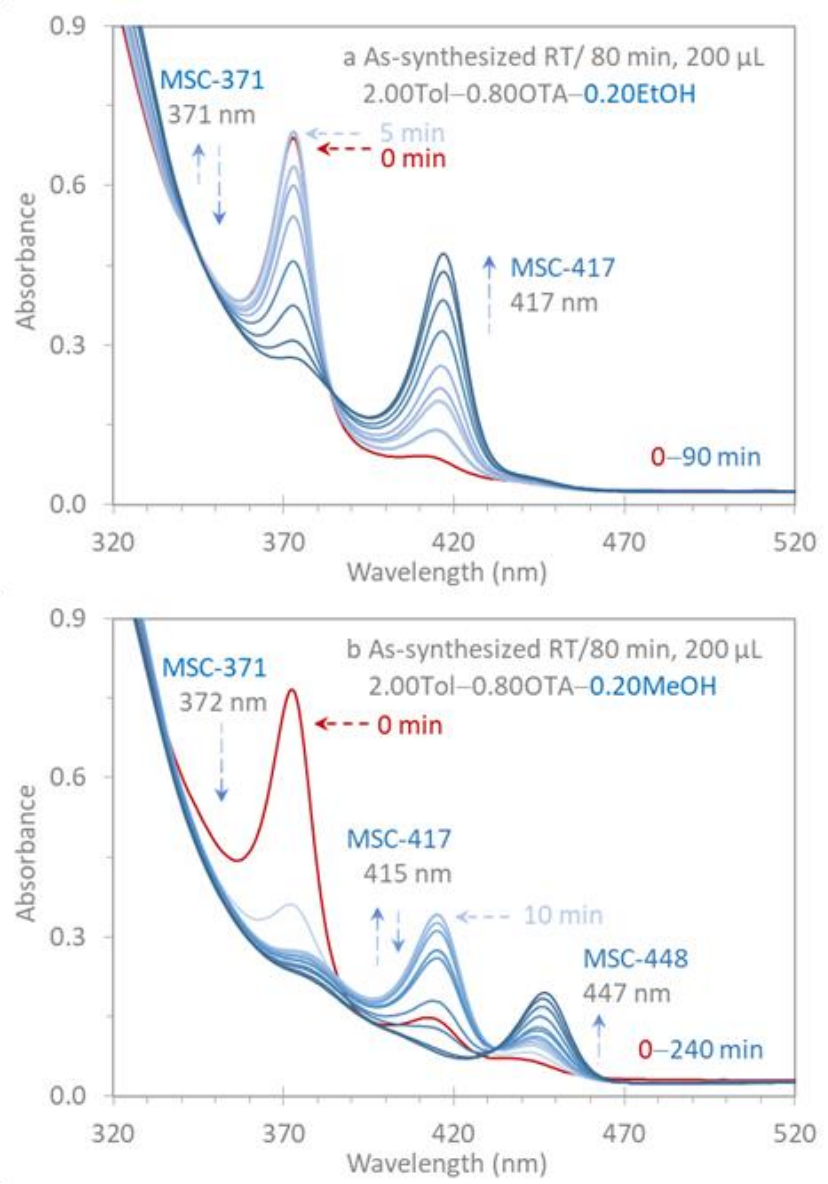

Figure S11. Optical absorption spectra collected from the same induction period sample (shown in Figure $\mathrm{S} 8$ ) in dispersion. The sample (200 $\mu \mathrm{L}$ ) was dispersed in two $3.00 \mathrm{~mL}$ mixtures of $2.00 \mathrm{~mL}$ Tol, $0.80 \mathrm{~mL} \mathrm{OTA}$, and $0.20 \mathrm{~mL} \mathrm{EtOH} \mathrm{(a)} \mathrm{or} \mathrm{MeOH} \mathrm{(b).} \mathrm{In} \mathrm{Part} \mathrm{a,} \mathrm{there} \mathrm{are}$ spectra collected at an interval of $5 \mathrm{~min}$ to $20 \mathrm{~min}$ and at 30, 40, 60, and $90 \mathrm{~min}$. MSC-371 changed little in the first 5 min and then decreased gradually, while MSC-417 increased monotonically with an isosbestic point at $\sim 383 \mathrm{~nm}$. In Part b, the spectra were collected with an interval of $5 \mathrm{~min}$ to $10 \mathrm{~min}$, an interval of $10 \mathrm{~min}$ to $60 \mathrm{~min}$, an interval of $30 \mathrm{~min}$ to 120 min, and then an interval of $60 \mathrm{~min}$ to $240 \mathrm{~min}$. MSC-371 decreased quickly, while MSC-417 reached its maximum at $10 \mathrm{~min}$. MSC-448 evolved continuously. One isosbestic point was at $\sim 431 \mathrm{~nm}$. MeOH promoted more than EtOH steps 3 and 6 in Scheme 1 from PC-371. 

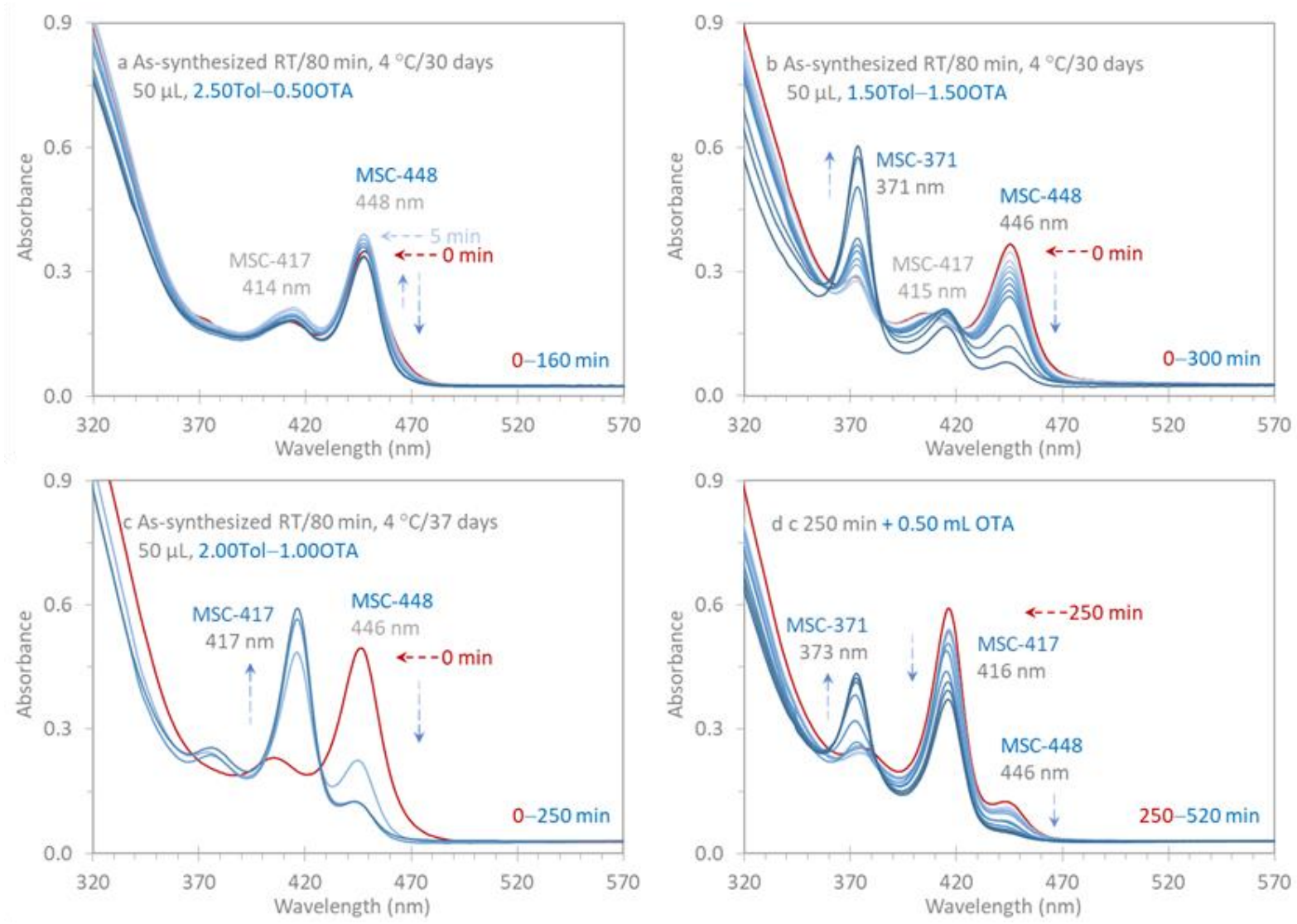

Figure S12. The same experiments shown in Figure 4, but with all optical absorption spectra collected. In Part a, there are nine spectra collected at 0, 5, 10, 20, 30, $40,80,120$, and 160 min. MSC-448 evolved immediately ( $0 \mathrm{~min}$, red trace) and reached its maximum at $5 \mathrm{~min}$; afterwards MSC-448 did not change much. In Part b, there are 12 spectra collected at 0, 5, $10,20,30,40,50,60,70,140,240$, and $300 \mathrm{~min}$. In Part c, the four spectra were collected at $0,150,230$, and $250 \mathrm{~min}$; after the addition of OTA (Part d), 10 spectra were collected at 255, 260, 270, 280, 300, 330, 390, 430, 470, and $520 \mathrm{~min}$. Both MSC-448 and MSC-417 started to decrease, companied with the notable growth of MSC-371. 

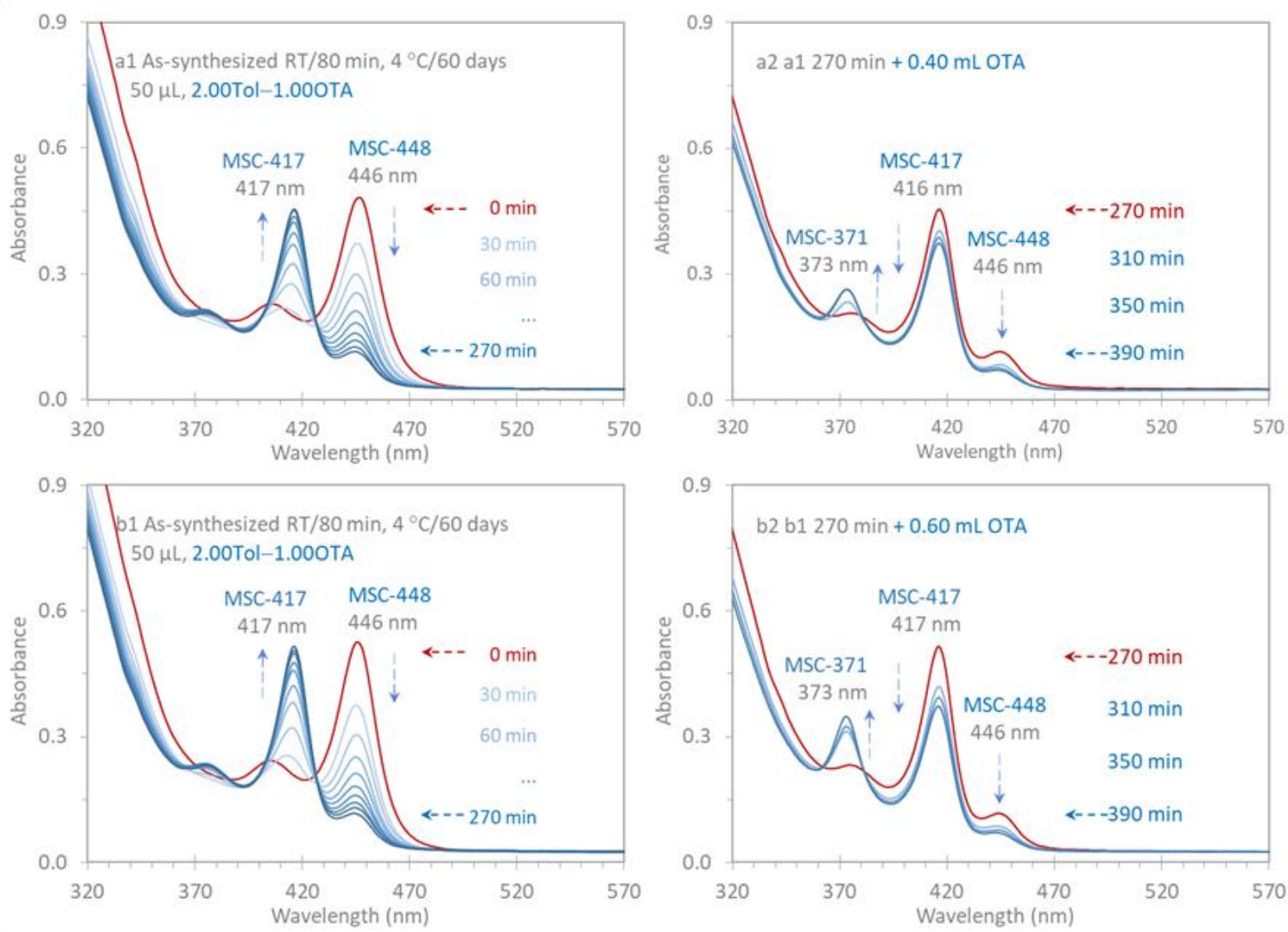

Figure S13. Optical absorption spectra collected from one induction period sample dispersed in Tol and OTA mixtures. The sample was from a room temperature reaction that proceeded for $80 \mathrm{~min}$ followed by an incubation at $4{ }^{\circ} \mathrm{C}$ for 60 days. Then, the sample ( $50 \mu \mathrm{L}$ ) was dispersed in two mixtures of Tol $(2.00 \mathrm{~mL})$ and OTA $(1.00 \mathrm{~mL})$. From each dispersion, 10 spectra were collected in $270 \mathrm{~min}$ ( $\mathrm{a} 1$ and b1) with an interval of $30 \mathrm{~min}$. MSC-448 formed immediately and then decreased, while MSC-417 evolved. After the addition of 0.40 (a2) and 0.60 (b2) $\mathrm{mL}$ of OTA, addition four spectra were collected from each dispersion during the period from 270 to $390 \mathrm{~min}$ with an interval of $40 \mathrm{~min}$. Both MSC-417 and MSC-448 decreased, while MSC-371 increased. Again, the larger amount of OTA pushes the equilibria to PC-371. 
Note S3 Additional discussion on the composition, configuration and size characterization of MSCs.

It has been challenging to characterize accurately the composition, size, and crystalline structure by conventical tools such as MS, TEM, and XRD. ${ }^{1-3}$

Based on a model proposed for the quantum confinement energy of semiconductor clusters by Brus, ${ }^{4,5}$

$$
\begin{aligned}
\mathrm{E}= & \left.\frac{\hbar^{2} \pi^{2}}{2 R^{2}}\left(\frac{1}{m_{e}}+\frac{1}{m_{h}}\right)-\frac{1.8 e^{2}}{\varepsilon_{2} R}+\frac{e^{2}}{R} \overline{\sum_{n=1}^{\infty} \alpha_{n}\left(\frac{S}{R}\right)^{2 n}} \text { (Equation } 12 \text { in Ref } 4\right), \text { where } \\
& \mathrm{E} \text { (a shift with respect to the bulk band gap) } \\
& =\text { band-edge energy of CdTe MSCs }- \text { CdTe bulk bandgap }(1.46 \mathrm{eV}) \\
& m_{e} \text { (electron effective mass) }=0.11 m_{0} \\
& m_{h} \text { (hole effective mass) }=0.35 m_{0} \\
& m_{0} \text { (electron mass) }=9.10956 \times 10^{-31} \mathrm{~kg} \\
& \varepsilon_{2} \text { (permittivity for CdTe bulk) }=9.42 \\
& e \text { (elementary charge) }=1.6 \times 10^{-19} \mathrm{C} \\
& \hbar \text { (reduced Planck constant) }=1.05457266(63) \times 10^{-34} \mathrm{~J} \cdot \mathrm{s} \\
& R \text { (radius of the nanocrystalline) } \\
& S \text { (a type of wave functions) } \\
& \left.\alpha_{n}=\frac{(\varepsilon-1)(n+1)}{\left[\varepsilon_{2}(\varepsilon n+n+1)\right]} \text { (Equation } 4 \text { in Ref } 4\right) \\
& \varepsilon=\frac{\varepsilon_{2}}{\varepsilon_{1}} \\
& \varepsilon_{1} \text { (medium of coefficient) }
\end{aligned}
$$

For the above equation which contains three parts, the first part contributes mainly to $\mathrm{E}$, which is the quantum confinement energy results from the localization of both electron and hole. The second and third parts are negligible.

We estimated the band-edge energy of the CdTe MSCs, which is about $3.35 \mathrm{eV}$ (MSC-371), $2.98 \mathrm{eV}$ (MSC-417), and $2.77 \mathrm{eV}$ (MSC-448).

Then, the diameter (2R) of the CdTe MSCs is accordingly around $3.1 \mathrm{~nm}$ (MSC-371), $3.4 \mathrm{~nm}$ (MSC-417), and $3.7 \mathrm{~nm}$ (MSC-448). These calculated values seem to be quite away from what the experimental data provided by SAXS, which are $1.9 \mathrm{~nm}$ (MSC-371), $2.8 \mathrm{~nm}$ (MSC-417), and $3.3 \mathrm{~nm}$ (MSC-448). ${ }^{3}$

Thus, a more advanced model is needed for the band-edge energy of semiconductor MSCs. We propose that additional parameters such as those related to cluster configuration and/or shape shall be considered.

(1) Wang, Y.; Zhou, Y.; Zhang, Y.; Buhro, W. E. Magic-Size II-VI Nanoclusters as Synthons for Flat Colloidal Nanocrystals. Inorg. Chem. 2015, 54, 1165-1177.

(2) Xie, L.; Shen, Y.; Franke, D.; Sebastian, V.; Bawendi, M. G.; Jensen, K. F. Characterization of Indium Phosphide Quantum Dot Growth Intermediates Using MALDI-TOF Mass Spectrometry. J. Am. Chem. Soc. 2016, 138, 13469-13472. 
(3) Liu, M.; Wang, K.; Wang, L.; Han, S.; Fan, H.; Rowell, N.; Ripmeester, J. A.; Renoud, R.; Bian, F.; Zeng, J.; Yu, K. Probing Intermediates of the Induction Period Prior to Nucleation and Growth of Semiconductor Quantum Dots. Nat. Commun. 2017, 8, 15467.

(4) Brus L. Electron-electron and Electron-hole Interactions in Small Semiconductor Crystallites: The Size Dependence of the Lowest Excited Electronic State. J. Phys. Chem. 1984, 80, 4403-4409.

(5) Brus L. Electronic Wave Functions in Semiconductor Clusters: Experiment and Theory. J. Phys. Chem. 1986, 90, 2555-2560.

Table S3. The synthetic condition for the induction period samples used.

\begin{tabular}{cccc}
\hline Figure & Condition & Storage $\left(-196{ }^{\circ} \mathrm{C}\right)$ & Incubation $\left({ }^{\circ} \mathrm{C}\right)$ \\
\hline Figure S4 & $60^{\circ} \mathrm{C} / 60 \mathrm{~min}$ & 0 day & 5 days \\
Figure S8 & $\mathrm{RT} / 80 \mathrm{~min}$ & 7 days & 0 day \\
Figure S11 & $\mathrm{RT} / 80 \mathrm{~min}$ & 9 days & 0 day \\
Figure 4ab, S12ab & $\mathrm{RT} / 80 \mathrm{~min}$ & 1 day & 30 days \\
Figure 4cd, S12cd & $\mathrm{RT} / 80 \mathrm{~min}$ & 1 day & 37 days \\
\hline Figure S13 & $\mathrm{RT} / 80 \mathrm{~min}$ & 1 day & 60 days \\
\hline
\end{tabular}


Table S4. Summary of the time interval ( $\mathrm{min}$ ) for spectra presented. In some figures not all collected spectra were shown.

\begin{tabular}{|c|c|c|c|c|c|c|c|c|c|c|c|}
\hline interval (min) & 1.5 & 5 & 10 & 20 & 30 & 40 & 50 & 60 & 70 & 80 & 100 \\
\hline Figure 2 & & $0-120$ & & & & & & & & & \\
\hline Figure S2 & & $0-60$ & & & & & & & & & \\
\hline Figure S3a & & $0-120$ & & & & & & & & & \\
\hline Figure S3bd & & & $0-120$ & & & & & & & & \\
\hline Figure S4a & & $0-30$ & $30-40$ & $40-80$ & $80-170$ & & $170-220$ & $220-280$ & & & \\
\hline Figure S4b & & $0-20$ & & & & & & & & & \\
\hline Figure S5 & & $0-60$ & & & & & & & & & \\
\hline Figure S6 & & & $0-120$ & & & & & & & & \\
\hline Figure S7a & $0-78$ & & & & & & & & & & \\
\hline Figure S7b & $0-24$ & & & & & & & & & & \\
\hline Figure S7c & $0-12$ & & & & & & & & & & \\
\hline Figure S8b & & $0-20$ & & & & & & & & & \\
\hline Figure S11a & & $0-20$ & $20-40$ & $40-60$ & $60-90$ & & & & & & \\
\hline Figure S11b & & $0-10$ & $10-60$ & & $60-120$ & & & $12-240$ & & & \\
\hline Figure S12a & & $0-20$ & $20-40$ & & & $40-160$ & & & & & \\
\hline Figure S12b & & $0-10$ & $10-70$ & & & & & $240-300$ & $70-140$ & & $140-240$ \\
\hline Figure S12d & & $0-10$ & $10-30$ & $30-50$ & $50-80$ & $140-220$ & $220-270$ & $80-140$ & & & \\
\hline Figure S13a1, b1 & & & & & $0-270$ & & & & & & \\
\hline Figure S13a2, b2 & & & & & & $270-390$ & & & & & \\
\hline
\end{tabular}

